\title{
Conditions for overall planarity in membranes: Applications to multicomponent membranes with lamellar morphology
}

\author{
F. J. Solis ${ }^{1(a)}$, C. M. Funkhouser ${ }^{2}$ and K. Thornton ${ }^{2}$ \\ ${ }^{1}$ Department of Integrated Natural Sciences, Arizona State University - Phoenix, AZ 85069, USA \\ ${ }^{2}$ Department of Materials Science and Engineering, University of Michigan - Ann Arbor, MI 48109, USA
}

received 21 October 2007; accepted in final form 5 March 2008

published online 15 April 2008

PACS 87.16.D- - Membranes, bilayers, and vesicles

PACS 87.16.A- - Theory, modeling, and simulations

\begin{abstract}
The coupling of geometric shapes and local composition in multicomponent membranes results in the formation of complex structures. These membranes can form structures that are planar at large length scales while retaining complex morphologies at smaller scales. We explicitly construct the overall planarity condition for membranes and identify it with the presence of an average tension at the membrane boundary with nonzero components only along the plane defined by the average height of the membrane. The explicit construction of the condition simplifies the analysis of morphologies of the planar membrane. We apply this method to the case of a bicomponent membrane with lamellar morphology. We determine the possible shapes of membranes, their stabilities and the thermodynamic equations of state satisfied by their intensive variables.
\end{abstract}

Copyright (c) EPLA, 2008

Two key features of multicomponent lipid membranes are their compositional and shape morphologies. Their interplay has important consequences for processes in biomembranes such as vesiculation and protein transport, and holds numerous possibilities for technological and biomedical applications. These features may be more easily explored when the overall, large-scale geometry of the system is planar; that is, when the membrane deviates from a flat background by only small distances. We call these membranes planar, for short. Several theoretical works have studied the static [1-3] and dynamic [4-7] properties of lamellar (striped) and caplet morphologies in planar membranes. Experimentally, the existence of these morphological phases has also been established [8,9]. The purpose of this work is to determine the precise conditions that lead to the existence of planar membranes and their implications for the mechanical and thermodynamical properties of such membranes. We find that planarity requires that the external forces at the membrane boundaries be confined to the background plane. While our key results are useful for all types of planar membranes, we focus in particular on the lamellar morphology, where the combination of the planar condition and the morphological symmetry allows us to analytically determine the shapes,

${ }^{(a)}$ E-mail: francisco.solis@asu.edu their stabilities, and the equations of state satisfied by their intensive variables.

We adopt the Helfrich model [10] to address the mechanical properties of the membranes. We assume that the components of the membrane form two distinct phases with sharp interfaces between them. We write the Helfrich free-energy functional for the system as [10]

$$
\tilde{F}=\sum_{i=1,2} \int \mathrm{d} A_{i}\left[\frac{1}{2} \Lambda_{i}\left(K-C_{i}\right)^{2}\right] .
$$

The phases 1 and 2 occupy areas $A_{1}$ and $A_{2}$, respectively. Each phase has different mechanical properties. The total area is $A=A_{1}+A_{2}$, and each of the areas can be composed of multiple domains. The bending rigidity and spontaneous curvature of each phase are denoted as $\Lambda_{i}$ and $C_{i}$, respectively. Following the notation of Capovilla and Guven [11], $K$ is the trace of the curvature tensor and is equal to twice the mean curvature of the surface. For simplicity, we treat only the symmetric case where the bending rigidities are equal, $\Lambda_{1}=\Lambda_{2}$. We assume the spontaneous curvatures have opposite signs (i.e., one concave and the other convex) and introduce the magnitude of their ratio $\beta=$ $-C_{2} / C_{1}$. We choose the phase labels and the orientation of the membrane so that $C_{1}>0$ and $C_{1} \geqslant\left|C_{2}\right|$ (i.e., $\beta \leqslant 1$ ). We nondimensionalize the free energy by 
measuring lengths in units of $1 / C_{1}$ and energies in units of $\Lambda$. These choices are equivalent to setting $\Lambda_{1}=\Lambda_{2}=1$, $C_{1}=1$, and $C_{2}=-\beta$. The thermal properties of our freeenergy functional are embedded in the implicit temperature dependence of the properties of the two phases.

We define a planar membrane as a thermodynamically stable macroscopic system with an average shape that extends along a plane (i.e., the $x-y$ plane) and has a finite extension onto the transversal direction (i.e., the $z$-direction). The geometric projection onto the $x$ - $y$ plane defines a (signed) projected area, while the projection onto the planes $x-z$ and $y-z$ is zero. Such states can be obtained by fully homogenous flat membranes, or those with microsegregated patterns. These patterns can be characterized by their ground-state symmetries, which in the binary case can only be lamellar and hexagonal caplet arrangements [1-3]. The analysis below will require that periodic boundary conditions be satisfied at the edges of the membrane; however, for sufficiently large membranes, this requirement should not affect the thermodynamic properties of the bulk. Thus we consider a sufficiently large membrane with regular boundaries that includes enough repetitions of the basic pattern, which can be thermodynamically described by a free energy $F$ that minimizes the Helfrich functional subject to the constraints on the values of four extensive variables: the areas occupied by each of the phases, $\left(A_{1}, A_{2}\right)$, the total length of the phase boundary (hereafter, interface), $B$, and the transversal area occupied by the membrane (or the projected area of the membrane onto the planar background), $D$. Thus, $F\left(A_{1}, A_{2}, B, D\right)=\min \tilde{F}$. The scheme in fig. 1 identifies the areas $A_{i}$ and the projection $D$ associated with a membrane patch with lamellar morphology. Analysis of the free energy as a function of the extensive variables is cumbersome, and it is simpler instead to use a thermodynamic potential, $\Psi$, conjugate to $F$ in all of these extensive variables:

$$
\Psi\left(\mu_{1}, \mu_{2}, \gamma, q\right)=F-\mu_{1} A_{1}-\mu_{2} A_{2}+\gamma B-q D .
$$

The conjugate variables are Lagrange multipliers that enforce the constraints on the values of the extensive variables. In addition, these variables have simple physical interpretations. We assume that the membrane is incompressible so that area changes are associated with absorption or desorption of molecules; thus, $\mu_{i}$ is the chemical potential of each segregated phase per unit area, measured with respect to the surrounding environment. These chemical potentials can be identified as the intrinsic surface tension for each phase $\left(\sigma_{i}=-\mu_{i}\right)$, although the effective surface tensions contain other contributions as discussed later. These two chemical potentials can be combined into a reference surface tension of the membrane $\sigma=-\left(\mu_{1}+\right.$ $\left.\mu_{2}\right) / 2$, and a chemical potential difference $\mu=\mu_{1}-\mu_{2}$. The interfacial length, $B$, is controlled by the line tension, $\gamma$. The term $\gamma B$ in eq. (2) is usually considered part of the mechanical free energy, but since it couples linearly to the interfacial length, it can be elevated to the status of
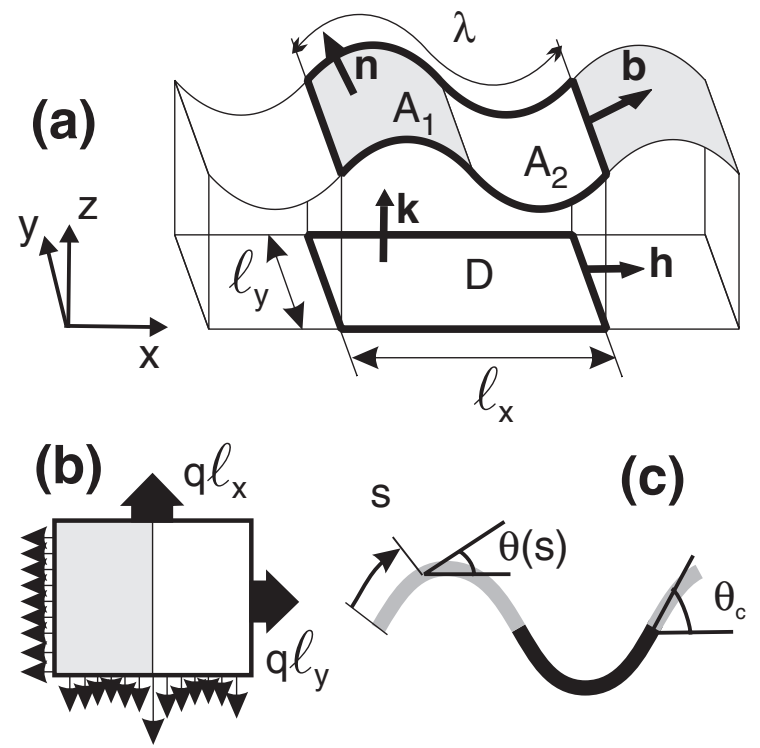

Fig. 1: Geometry of the lamellar morphology: (a) a rectangular patch of a planar binary membrane with strongly segregated phases shown in grey and white; (b) a sketch of a distribution of external tensions required for planarity at the boundaries of the projection of a rectangular subsection; the top and right edges are acted on by the total forces; (c) a parametrized crosssection, with local varying angles $\theta(s)$, and contact angle at the boundary between regions $\theta_{c}$.

a thermodynamic conjugate variable. The final Legendre transform with respect to the projected area introduces the lateral tension, $q$, which we discuss in detail below. We note that these conjugate variables can be defined as derivatives of the mechanical free energy considered as a function of the extensive variables $F\left(A_{1}, A_{2}, B, D\right)$ :

$$
\mu_{i}=\frac{\partial F}{\partial A_{i}}, \quad \gamma=-\frac{\partial F}{\partial B}, \quad q=\frac{\partial F}{\partial D}
$$

While the fundamental definitions of the conjugate variables $\mu_{i}, \gamma$, and $q$, are based on the free energy $F$ that depends on the extensive variables, the thermodynamic potential we have defined is a function of the intensive variables conjugate to the extensive quantities, i.e., $\Psi=\Psi\left(\mu_{i}, \gamma, q\right)$. These variables can also be considered as conjugate to four ratios: two area fractions $\phi_{i}=A_{i} / A$, the boundary length density $b=B / A$, and the projected area ratio $d=D / A$. Finally, we define the mechanical free-energy density $f=F / A$. All of these quantities are averaged over a unit cell.

The requirement that the membrane should have a projected area $D$ onto the $x-y$ plane and zero on planes perpendicular to it constrains its possible conformations. Let us now examine in more detail this planarity constraint. We consider a membrane patch of area $\mathrm{d} A$ with a unit normal vector $\mathbf{n}$. The patch is projected onto a plane with unit normal $\mathbf{k}$, and the projected area is $\mathrm{d} D=\mathbf{k} \cdot \mathbf{n} \mathrm{d} A$. Therefore, the term $-q D$ in the potential $\Psi$ corresponds to the inclusion of a term of the form 
$-\int q \mathbf{k} \cdot \mathbf{n} \mathrm{d} A$ in the corresponding variational functional $\tilde{\Psi}$, and constrains the projected area of the membrane. This term leads, in principle, to new contributions to the shape equations, boundary conditions and stress tensor. To derive these terms, we apply the methods and results from refs. $[11,12]$. The notations adopted from the references are given below. Each point on the membrane is labelled by two-dimensional coordinates $\left(u^{1}, u^{2}\right)$ and occupies a position $\mathbf{X}\left(u^{a}\right)$ in three-dimensional space, where $a=1,2$. Bold fonts are used throughout the paper to denote three-dimensional vectors. This representation defines a tangent vector base $\mathbf{e}_{a}=\partial_{a} \mathbf{X}=\partial \mathbf{X} / \partial u^{a}$, a gradient operator $\nabla_{a}$ that reduces to a partial derivative on scalars but acts in a more complicated manner on vectors, and the metric tensor and its inverse, $g_{a b}, g^{a b}$, where $g_{a b}=\mathbf{e}_{a} \cdot \mathbf{e}_{b}, g^{a b} g_{b c}=\delta_{c}^{a}$. We use Einstein's summation convention and standard upper and lower indices for contravariant and covariant vectors, respectively.

Our basic results are as follows:

i) The new potential density term $-q \mathbf{k} \cdot \mathbf{n d} A$ does not modify the shape equation. A general shape variation takes the form $\delta \mathbf{X}=\phi^{a} \mathbf{e}_{a}+v \mathbf{n}$ for arbitrary infinitesimal parameters $\phi^{a}$ and $v$. We rewrite the variation of our term as a total derivative: $\delta(-q \mathbf{k} \cdot \mathbf{n} \mathrm{d} A)=\nabla_{a} U^{a} \mathrm{~d} A$, with $U^{a}=-q\left(\phi^{a} \mathbf{k} \cdot \mathbf{n}-g^{a b} v \mathbf{k} \cdot \mathbf{e}_{b}\right)$. A total derivative can be integrated out to a boundary term and thus it does not contribute to any bulk equations, including the local shape equation.

ii) For a system where we require the boundary itself to be in equilibrium, boundary contributions due to a normal shape deformation, $\delta \mathbf{X}$, must cancel among each other. We find that this new term, stemming from the planar condition, manifests as an average equilibrating term that compensates the boundary contributions of the Helfrich free energy in eq. (1). Given a unit vector $\mathbf{b}=b^{a} \mathbf{e}_{a}$ normal to a smooth boundary and tangent to the membrane, the integral of a divergence in a manifold can then be recast as a boundary integral: $\int_{M} \nabla_{a} U^{a} \mathrm{~d} A=\int_{\partial M} U^{a} b_{a} \mathrm{~d} t$, where $\mathrm{d} t$ stands for an infinitesimal length segment of the boundary. In other words, the planarity constraint determines a set of boundary conditions for the membrane in general.

iii) At a smooth cut or boundary of the membrane we find a three-dimensional force density vector $\mathbf{f}$. This force density depends on the direction of the cut, which on the boundary is specified by the boundary normal $\mathbf{b}=b^{a} \mathbf{e}_{a}$. The force density is related to the stress tensor of the membrane $\mathbf{f}^{a}=\left[\mathbf{f}^{1}, \mathbf{f}^{2}\right]$ through $\mathbf{f}=\mathbf{f}^{a} b_{a}$. This stress tensor consists of two three-dimensional vectors (indicated by bold face), one for each value of the index $a$ (see [11-13]). The tensor can be written as $\mathbf{f}^{a}=f_{\|}^{a b} \mathbf{e}_{b}+f_{\perp}^{a} \mathbf{n}$, where $f_{\|}^{a b}$ and $f_{\perp}^{a}$ are the tangential and normal components of the tensor, respectively. Each term of the variational potential gives rise to a contribution to this tensor. We have shown that a variation $\delta \mathbf{X}$ normal to the surface takes the form $-\nabla_{a}\left(\delta \mathbf{X} \cdot \mathbf{f}^{a}\right)$. This expression allows us to identify the stress-tensor contribution of our constraint $\mathbf{f}_{\mathrm{D}}^{a}$, and we obtain the final net boundary force density due to the constraint $\mathbf{f}_{\mathrm{D}}=\mathbf{f}_{\mathrm{D}}^{a} b_{a}$ :

$$
\mathbf{f}_{\mathbf{D}}=q \mathbf{k} \times(\mathbf{b} \times \mathbf{n}) .
$$

This form of the boundary force contribution has a very clear property: it is always in the background plane, perpendicular to the boundary of $D$, and pointing outward of $D$, as is evident from the cross product of $\mathbf{k}, \mathbf{b}$, and $\mathbf{n}$.

iv) Let us now consider the stress tensor associated with the planar projection of the membrane, instead of the membrane itself. The planar projection has a boundary unit normal $\mathbf{h}=(\mathbf{k} \times(\mathbf{b} \times \mathbf{n})) /|\mathbf{k} \times(\mathbf{b} \times \mathbf{n})|$ (parallel to the projection plane), and the infinitesimal length along the projected boundary is $\mathrm{d} \ell$. We can then recast the constraint contribution to the net force on a boundary segment as $\mathbf{f}_{\mathbf{D}} \mathrm{d} t=q \mathbf{h} \mathrm{d} \ell$. Therefore, the stress tensor contribution $\mathbf{f}_{\mathrm{D}}^{a}$ can also be interpreted as the stress tensor on the projected plane. The effective stress tensor $T_{\alpha \beta}^{D}$ in this plane, using Cartesian coordinates, is $T_{\alpha \beta}^{D}=q \delta_{\alpha \beta}$. This tensor is homogeneous and isotropic. External forces applied at the boundary of a membrane patch that satisfy this structure can therefore be used to create a planar membrane, establishing the planar constraint.

v) The equilibrium of a large but finite membrane requires in general the application of inhomogeneous distributions of forces at the boundary, as sketched at the bottom boundary of fig. 1(b). The force created by the planar constraint cannot locally equilibrate the forces required for equilibrium of the membrane. From a thermodynamic point of view, however, we are concerned only with average quantities over scales larger than the characteristic morphological length (e.g., the repetition length $\lambda$ in the lamellar morphology). Therefore, states in thermodynamic equilibrium satisfy $\left\langle\mathbf{f}^{a}\right\rangle=\mathbf{0}$, where the braces indicate an average over a large boundary segment. The contribution of the planarity constraint $\mathbf{f}_{D}^{a}$ then serves to compensate all other contributions. Since this tensor lies within the projected plane, the inclusion of the constraint $-q D$ requires that the average stress tensor of the remaining contributions also be confined in the same plane.

vi) Let us consider the deformation of a membrane patch with rectangular projection of dimensions $L_{x}$ and $L_{y}$ that satisfies periodic boundary conditions. The energy of deformation is given by the integration over the boundary of the membrane of contributions due to the stress tensor and the torque density $H^{a b}$ (see ref. [12]). This density is $-b_{a}\left(\mathbf{f}^{a} \cdot \delta \mathbf{X}-H^{a b} \mathbf{e}_{b} \cdot \delta \mathbf{n}\right)$. When the dimensions of the patch are large enough, the stress tensor term is zero on average thanks to the inclusion of the planarity constraint. When the periodic boundary conditions are respected by the deformation, the torque contributions at opposite points of the boundary cancel each other. We therefore have full equilibrium of the membrane against large-scale deformations. We can now construct a membrane patch of arbitrary size $A$ by extending a smaller size membrane. When the extension is sufficiently regular, any change in the thermodynamic potential due to a small deformation 
is zero on the average: that is, $\delta \Psi=\int \mathbf{f}^{a} b_{a} \cdot \delta \mathbf{X} \mathrm{d} s=0$ since $\left\langle\mathbf{f}^{a}\right\rangle=\mathbf{0}$ and $b_{a}$ and $\delta \mathbf{X}$ are uniform in deformations that maintain a rectangular shape with periodic boundary conditions. Therefore, the potential is constant. Since this potential is an extensive quantity, it takes the value of zero for a system of zero area. We thus conclude that the potential is identically zero $\Psi \equiv 0$ at all areas; we refer to a potential with such properties as a null potential.

vii) Finally, we examine the thermodynamic role of the lateral tension term $-q D$ in eq. (2). Because this term accounts for the work done by external forces applied to the boundary, the potential $\Psi$ describes finite but large systems using a thermodynamic ensemble based on the intensive variables $\mu_{i}, q$ and $\gamma$. When the values of the intensive variables are specified, the absolute values of the extensive variables $\left(A_{i}, B, D\right)$ are only determined up to a scale factor; only their ratios are specified. A basic theorem of thermodynamics [14] indicates then that the intensive variables are not independent and that an equation of state relates them. This equation can be obtained from the condition $\Psi=0$, which was derived above, and the explicit form will be discussed later.

We apply these results i)-vii) to the case of lamellar morphologies sketched in fig. 1. As shown in fig. 1(c), the coordinate $s$ measures the arc length along a transversal section. The lengths of the repeated sections of each species are $s_{1}$ and $s_{2}$, and we define $\lambda=s_{1}+s_{2}$; because of the continuous translational symmetry of the system in the $y$-direction, we have $s_{1} / \lambda=\phi$. The local shape is measured by means of an angle $\theta$ with respect to the projection plane. The crucial terms in the potential are the projection, $\mathbf{k} \cdot \mathbf{n}=\cos \theta$, and the trace of the curvature tensor $K=\theta^{\prime}$, where the prime indicates differentiation with respect to the arc length $s$. For a large rectangular region of dimensions $L_{x} \times L_{y}$, the variational potential $\tilde{\Psi}$ to be minimized in order to obtain $\Psi$ is given by

$$
\frac{\tilde{\Psi}}{L_{y}}=\sum_{i=1,2} \int \mathrm{d} s\left[\frac{\left(\theta^{\prime}-C_{i}\right)^{2}}{2}-\mu_{i}-q \cos \theta\right]+2 n \gamma,
$$

where the last term of the functional is the expression of the interfacial boundary length $B$ in terms of the number of lamellar repetitions per unit length, $n$. Note that we have $d=D / A=L_{x} / S$, where $S$ is the total arc length in a cross-section. Instead of considering direct variations with respect to the coordinate field $\mathbf{X}$, we determine more easily interpreted equilibrium conditions by direct variation of the angle variable $\theta$. This technique has been employed previously, and many aspects of the solutions are known [15-17]; however, its thermodynamical implications have not yet been explored.

The shape equation of the membrane is obtained from variation of the potential $\tilde{\Psi}$ with respect to $\theta$. We decompose the variation into a bulk term, $V_{k}$, and a boundary term, $V_{b}$, as: $\delta \tilde{\Psi} / L_{y}=\int \mathrm{d} s\left(V_{k} \delta \theta+\partial_{s}\left(V_{b} \delta \theta\right)\right)$. The bulk equilibrium is obtained by setting $V_{k}$ to zero:

$$
\theta^{\prime \prime}-q \sin \theta=0 \text {. }
$$

This equation is valid at all points within the homogeneous regions. At interfaces, where two homogeneous regions meet, the boundary contributions $V_{b}$ from each homogeneous region should cancel each other to make the net variation of the potential zero. This leads to the following condition at the interfaces:

$$
\theta_{1}^{\prime}-C_{1}=\theta_{2}^{\prime}-C_{2}
$$

Another set of equations can be obtained by considering a local extension of the membrane. To do so, one can introduce a variable $p$ that parametrizes the integrand of $\tilde{\Psi}$. This introduces a Jacobian $j=\mathrm{d} s / \mathrm{d} p$, and we have $\theta^{\prime}=(1 / j) \mathrm{d} \theta / \mathrm{d} p$. Variation of the free energy with respect to the Jacobian $j$ is physically equivalent to the local extension. Carrying out the variation and evaluating at $j=1$, we obtain

$$
-\frac{1}{2} \theta^{\prime 2}+\frac{1}{2} C_{i}^{2}-\mu_{i}-q \cos \theta=0
$$

where $i$ specifies the phases. This result, eq. (8), is in fact an integration of eq. (6). In addition, by taking a derivative of eq. (6) with respect to the arc length, and then using eq. (8) to eliminate the cosine, we obtain $\theta^{\prime \prime \prime}+\theta^{\prime} 3 / 2+$ $\left(-\mu_{i}+C_{i}^{2} / 2\right)=0$. With suitable identifications, such as $\theta^{\prime \prime \prime}$ being the Laplacian of the curvature, this is in fact the standard shape equation of the membrane. Thus our formulation, which considers the lateral tension and the planarity condition, is consistent with the shape equations derived in a classical manner (for example, by examination of balances of forces and torques $[18,19]$ ). We note that the lateral tension does not appear explicitly in the classical form of the shape equation.

The shape of the membrane can be obtained, for example, by solving eq. (8), which can be written in terms of the Jacobi amplitude function $a m$ :

$$
\theta(s)= \pm a m\left(\left(s-s_{k}\right) r_{i}, \frac{4 q}{r_{i}^{2}}\right),
$$

with $r_{i}=\sqrt{-2 \mu_{i}+C_{i}^{2}-2 q}$. In this expression, $s_{k}$ is an arc length value at which the angle is zero. The sign in the right-hand side of the equation is chosen according to the spontaneous curvature of the region: negative for phase 1 , (with $C_{1}=1$ ) and positive for phase 2 .

The boundary conditions, eq. (7), lead to a unique value of the contact angle, $\theta_{c}$, defined in fig. $1(\mathrm{c})$, which is given by

$$
q \cos \theta_{c}=-\frac{\mu}{2(1+\beta)^{2}}\left(\mu-1+\beta^{2}\right)+\sigma .
$$

We join regions of phase 1 that span angles from $\theta_{c}$ to $-\theta_{c}$ forming a convex shape, and match a region of phase 2 with angles from $-\theta_{c}$ to $\theta_{c}$. Together, they create a basic cell of the periodic solution. Other solutions exist in which the values of the angle exceeds $2 \pi$; these solutions are discarded as they lead to unphysical self-intersections of the membrane. The explicit form of the solution for 

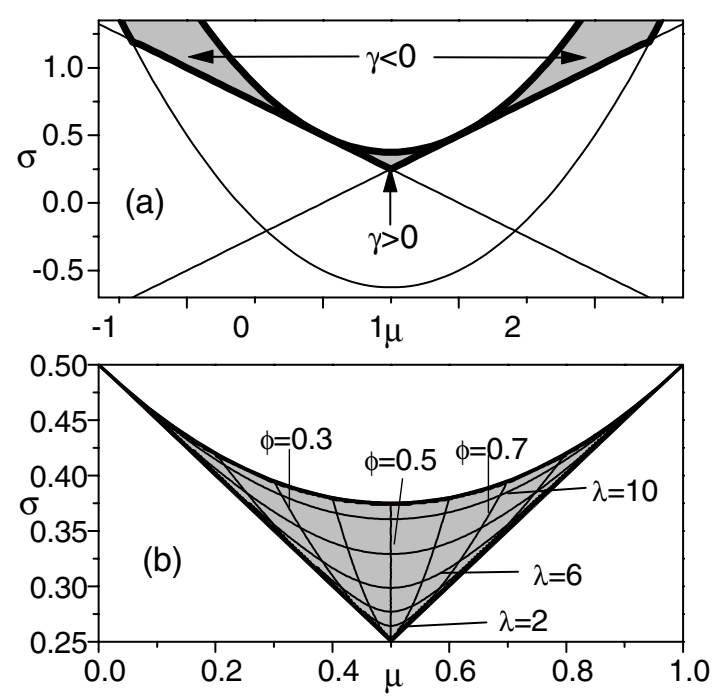

Fig. 2: In (a) the physical regions in the $\sigma$ - $\mu$ plane are shaded. The straight lines and parabolas are limiting lines of the regions allowed by conditions/eqs. (11) and (12). The lateral tension is $q=0.5$ and the curvature ratio is $\beta=0$. Panel (b) shows a detailed view of the physical region with $\gamma>0$ of panel (a). We show isocontour lines for the area fraction $\phi$ and periodicity $\lambda$.

the angle variable can be used to obtain the local height, contour lengths and horizontal projections as well. These solutions have also been recovered (using a fixed-surfacetension, fixed-periodicity ensemble) in recent simulations by our group [6].

The solutions described above must obey a number of restrictions in their allowed parameters. First, we note that the square of the spontaneous curvature in the shape equation, eq. (8), requires the remaining terms to add to a positive quantity. As we will show later, $q>0$ for stable solutions. Thus, considering the limiting case at $\cos (\theta)=1$, we obtain

$$
\min \left(\theta^{\prime 2}\right)=C_{i}^{2}-2 \mu_{i}-2 q \geqslant 0
$$

within each homogeneous region $i=1,2$. In addition, the contact angle must be physical,

$$
-1 \leqslant \cos \left(\theta_{c}\right) \leqslant 1
$$

thus restricting the right-hand side of eq. (10).

We note that $\gamma$ does not appear in the solutions above since, due to the symmetry of the lamellar system, its energy density is concentrated at the interfaces and does not modify the bulk equation nor the boundary conditions. To examine the role of $\gamma$ we use the equation of state, $\Psi=0$. After some substitutions in eq. (2), and division by the total area $A$, the equation of state can be expressed as

$$
f+\sigma-\mu\left(\phi-\frac{1}{2}\right)-q d+\frac{2 \gamma}{\lambda}=0 .
$$

The solutions constructed above allow determination of $f$, $\phi, d$ and $\lambda$ as a function of $\sigma, \mu$, and $q$ only, and therefore, $\gamma$ can simply be evaluated using this expression.
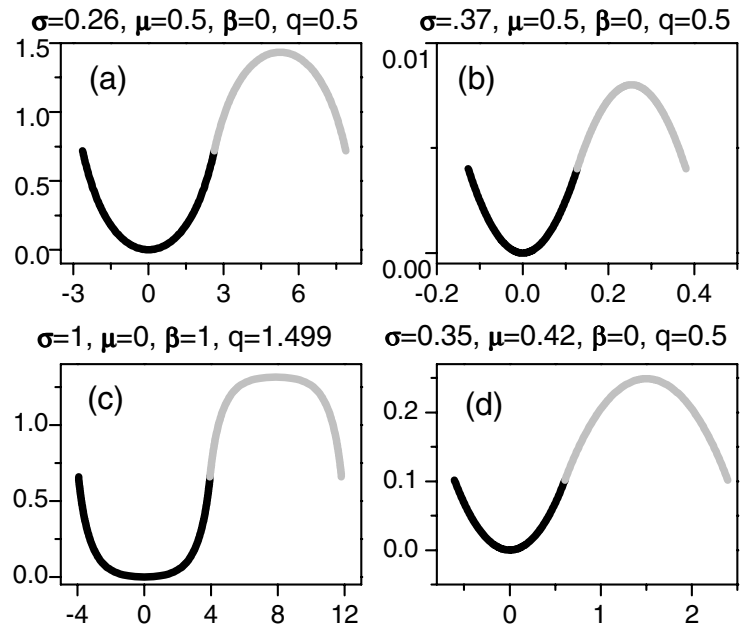

$\sigma=1.37, \mu=1.75, \beta=0, q=0.5$
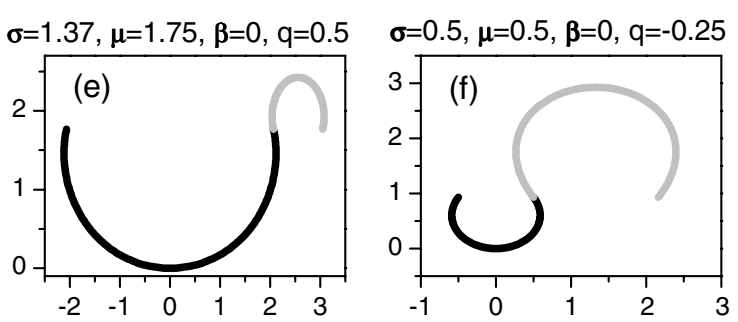

Fig. 3: Cross-sections of repeating shape patterns. Parameters as shown in headers.

We can now present the properties of the system in terms of three of the four intensive variables $\mu_{1}, \mu_{2}, \gamma$, and $q$. It is easiest to eliminate $\gamma$ as an independent variable using the equation of state and then study the behavior of dependent variables as functions of $\sigma$ and $\mu$ while keeping $q$ constant. In this slice of parameter space, realizable points are bounded by four boundaries determined by eqs. (11) and (12). These boundaries form, for generic values of the tension $q$, three different regions as shown in fig. 2(a), for the particular case of $q=0.5$ and $\beta=0$. Two points of contact divide the regions; their explicit coordinates can be obtained solving for the intersection of the boundaries defined by eqs. (11) and (12). These points have coordinates $\sigma=q-\beta / 2$, and $\mu=1+\beta,-\beta(1+\beta)$. Inspection of the equation of state reveals that the two side regions have $\gamma<0$, and thus are unphysical. We note, however, that solutions in these regions exhibit overhangs (see fig. 3(e,f)), which are important features observed in the dynamics of certain surfactant films [20]. In fig. 2(b) we expand the physical region with $\gamma>0$ and show isocontours of $\phi$ and $\lambda$. Isocontours for the mechanical free-energy density $f$ and the equilibrium line tension $\gamma$ have similar shapes to those of $\phi$ and $\lambda$, respectively.

Sample shapes obtained from our solutions are shown in fig. 3 for the parameter values shown in each frame. Panel (a) shows a very regular, generic solution with $\phi=0.5$. Panels $(b, c)$ show that, while the characteristic length of the system is $1 / C_{1}=1$, the lamella spacing and height of the ripple can be (b) smaller than or (c) larger than 
the characteristic length. Panel (d) has an asymmetric area fraction $\phi=0.4$. Panels (e,f) belong to the unphysical regions $(\gamma<0)$ and show overhangs. It can be shown that when the phase fraction is symmetric, $\phi=0.5$, and the bending rigidities are equal, the shapes of the two phases are mirror images even when their intrinsic curvatures are not equal, as in $(\mathrm{a}, \mathrm{b})$.

Let us now consider the stability of these solutions. Taking the second variation of the potential with respect to the local angle, we obtain a quadratic functional on the first-order variations. We write the result in terms of a Schrödinger-type operator, $\delta^{2} \Psi=\int \mathrm{d} s \delta \theta \hat{H} \delta \theta$ with

$$
\hat{H}=-\frac{\mathrm{d}^{2}}{\mathrm{~d} s^{2}}+q \cos \theta(s)
$$

The stability condition $\delta^{2} \Psi \geqslant 0$ has a direct solution; since the operator $\hat{H}$ is Hermitian, its eigenvalues, $\xi_{i}$, must be positive. Conversely, the system is unstable if at least one of the eigenvalues becomes negative. Instead of this direct approach, it is also possible to examine instabilities of the system by finding deformations $\delta \theta$ that render the integral negative [21]. Using this latter method we can show that planar membranes under (strong) compression $q<0$ are in general unstable. For large values of the compression force, the lateral tension term in the operator, $q \cos \theta$, becomes large and negative, and any trial variation centered around a position $s$ with $\theta=0$ creates an instability. This is similar to the classical result of columns under compression [22]. Due to this instability, we do not consider compressive states in our phase diagram, as mentioned earlier.

The membrane under tension is typically found to be stable. Instabilities can appear only when the term $q \cos \theta$ becomes negative in a region. This occurs when there are overhangs in the shape, where $\cos \theta<0$, so that locally the membrane is under compression. It can be shown that such shapes occur only in regions of the phase diagram that correspond to negative line tension, $\gamma<0$, which are already unstable (as $\gamma<0$ causes complete mixing). In the central region of the phase diagram, with $\gamma>0$, overhangs are not possible, and the system is always stable.

As we have shown, the thermodynamics and stability of planar membranes are greatly illuminated by the explicit introduction of the planarity constraint and, as in other recent works, by the consideration of forces and stresses $[13,18,19]$. The Lagrange multiplier $q$ associated with the constraint is interpreted as an external tension acting on the boundary of the membrane and also as the thermodynamic variable conjugate to the projected area of the membrane. We note that while we have exploited in detail these relations in the context of the lamellar morphologies, they should also be useful for caplet morphologies, and will enable the reinspection of the phase diagrams obtained in recent works $[1-3]$.

The authors thank J. GUVEN for helpful discussions on this subject. CMF acknowledges the generous support from NSF through her Graduate Research Fellowship. The authors also acknowledge the financial support provided by UM OVPR.

\section{REFERENCES}

[1] Gòz̀dz̀ W. T. and Gompper G., Europhys. Lett., 55 (2001) 587.

[2] Harden J. L., MacKintosh F. C. and Olmsted P. D., Phys. Rev. E, 72 (2005) 011903.

[3] Ni D., Shi H.-J., Yin Y.-J. and Niu L.-S., J. Biol. Phys., 32 (2006) 369.

[4] Reigada R., Buceta J. and Lindenberg K., Phys. Rev. E, 71 (2005) 051906.

[5] Kunsenok O. and Balazs A. C., Phys. Rev. E, 75 (2007) 051906.

[6] Funkhouser C. M., Solis F. J. and Thornton K., Phys. Rev. E, 76 (2007) 011912.

[7] Sample C. and Golovin A. A., Phys. Rev. E, 76 (2007) 031925.

[8] Keller S. L. and McConnell H. M., Phys. Rev. Lett., 82 (1999) 1602.

[9] Rozovsky S., Kaizuka Y. and Groves J. T., J. Am. Chem. Soc., 127 (2005) 36.

[10] Helfrich W., Z. Naturforsch. C, 28 (1973) 693.

[11] Capovilla R. and Guven J., J. Phys. A, 35 (2002) 6233.

[12] Guven J., J. Phys. A, 37 (2004) L313.

[13] Fournier J.-B., Soft Matter, 3 (2007) 883.

[14] Callen H. B., Thermodynamics and an Introduction to Thermostatistics (Wiley, New York) 1985.

[15] Kawakatsu T., Andelman D., Kawasaki K. and Taniguchi T., J. Phys. II, 3 (1993) 971-997.

[16] Ou-Yang Z.-C., LiU J.-X. and XIE Y.-Z., Geometric Methods in the Elastic Theory of Membranes in Liquid Crystal Phases (World Scientific, Singapore) 1999.

[17] Capovilla R., Guven J. and Santiago J. A., J. Phys. A, 36 (2003) 6281.

[18] Müller M. M., Deserno M. and Guven J., Phys. Rev. $E, 72$ (2005) 061407.

[19] Deserno M., Müller M. M. and Guven J., Phys. Rev. E, 76 (2007) 011921.

[20] Diamant H., Witten T. A., Gopal A. and Lee K. Y. C., Europhys. Lett., 52 (2000) 171.

[21] Ballentine L. E., Quantum Mechanics: A Modern Development (World Scientific, River Edge, NJ) 1998, sect. 10.

[22] Landau L. D. and Lifshitz E. M., Theory of Elasticity (Pergamon Press, New York) 1986. 